\title{
Flare Hybrids
}

\author{
M. Tomczak ${ }^{1} \cdot$ P. Dubieniecki ${ }^{1}$
}

Received: 9 December 2014 / Accepted: 13 April 2015 / Published online: 29 April 2015

(C) The Author(s) 2015. This article is published with open access at Springerlink.com

\begin{abstract}
On the basis of the Solar Maximum Mission observations, Švestka (Solar Phys. 121, 399, 1989) introduced a new class of flares, the so-called flare hybrids. When they start, they look like typical compact flares (phase 1), but later on, they look like flares with arcades of magnetic loops (phase 2). We summarize the characteristic features of flare hybrids in soft and hard X-rays as well as in the extreme ultraviolet; these features allow us to distinguish flare hybrids from other flares. In this article, additional energy release or long plasma cooling timescales are suggested as possible causes of phase 2 . We estimate the frequency of flare hybrids, and study the magnetic configurations favorable for flare hybrid occurrence. Flare hybrids appear to be quite frequent, and the difference between the lengths of magnetic loops in the two interacting loop systems seem to be a crucial parameter for determining their characteristics.
\end{abstract}

Keywords Flares $\cdot$ X-Rays $\cdot$ Magnetic fields

\section{Introduction}

There are no two identical flares, but it is useful to classify flares following some schemes. The most commonly accepted classification was introduced by Pallavicini, Serio, and Vaiana (1977) based on soft X-ray images obtained by the S-054 experiment onboard Skylab. The authors proposed two separate classes of events, namely compact flares (class 1) and flares occurring in large and diffuse systems of loops (class 2). They found that the separation is supported by the different values of several physical parameters such as height, volume, energy density, and characteristic times of rise, decay, and duration. They also perceived that flares of class 1 are located very low in active regions and, in contrast to flares of class 2 , do not appear to be associated with coronal mass ejections (CMEs) and prominence eruptions or activations.

Solar and Stellar Flares: Observations, Simulations, and Synergies

Guest Editors: Lyndsay Fletcher and Petr Heinzel

M. Tomczak

tomczak@astro.uni.wroc.pl

1 Astronomical Institute, University of Wrocław, ul. Kopernika 11, 51-622 Wrocław, Poland 
The division into two classes opposed to each other by contradiction is called dichotomy, therefore we call the classification of Pallavicini, Serio, and Vaiana (1977) flare dichotomy for short. The flare dichotomy has been supported by several classifications, e.g. impulsive $v s$. long-duration flares, single-loop vs. arcade flares, confined $v s$. eruptive flares, or twofootpoint $v s$. two-ribbon flares.

Beyond any doubt, the division into two classes is very rough; therefore, it is possible that observed flares can share features of both class 1 and class 2. Švestka (1989) introduced for them the term flare hybrids. What does a flare hybrid look like? Its evolution can be divided into two phases: during phase 1 it looks like a flare of class 1, and during phase 2 it looks like a flare of class 2. Recalling a private communication with Cornelius de Jager, Švestka (1989), discussed that a flare of class 1 may trigger a flare of class 2 . He also investigated the process that causes the magnetic field to open and thus start a flare of class 2 .

Further observations made with many instruments at different wavelengths have provided a more complete picture of flare hybrids. In Section 2 we present characteristic features of flare hybrids in soft X-ray (SXR), hard X-ray (HXR), and extreme-ultraviolet (EUV) ranges. In Section 3 we lay down some rules for the frequency of occurrence. The magnetic configuration suggested for flare hybrids is described in Section 4. In Section 5 we propose the most likely scenario for a flare hybrid.

\section{Observations}

\subsection{Soft X-Rays}

In Figure 1 we present an example of the flare hybrid, SOL1992-11-05T06:22 (M2.0), observed by the Soft X-ray Telescope (SXT: Tsuneta et al., 1991) onboard the Yohkoh satellite. We show the three SXR images made with the AlMg filter during phase 1 (Figure 1a), during phase 2 (Figure 1c), and in the intermediate time (Figure 1b). During phase 1 the SXR emission of the flare is clearly dominated by a small $\left(h \approx 10^{4} \mathrm{~km}\right)$ system of bright loops. Later on, a higher magnetic arcade $\left(h \approx 5 \times 10^{4} \mathrm{~km}\right)$ is seen in SXRs during phase 2 . In each image we mark the borders of two areas, 1 and 2, within which the SXR signal was measured. We plot the light curves for these areas and the total signal from all the images in Figure 1d. The time gaps in the light curves are mainly caused by the satellite night. The light curves for areas 1 and 2 are different, but together they have a double-peaked shape. The same double-peaked light curve was recorded by the GOES satellite (Figure 1e), where the time interval of the satellite night is also marked.

However, a double-peaked GOES light curve cannot be considered as a typical signature of a flare hybrid. Another example of a flare hybrid, SOL1992-09-09T18:03 (M1.9), observed by the SXT, is shown in Figure 2. The panels in the figure are organized in the same way as in Figure 1. The difference is due to the choice of SXT filter, Al12. The evolution of the flare presented in Figure 2 is very similar to that presented in Figure 1. During phase 1 the SXR emission of the flare is dominated by a smaller area 1 around a system of lower loops, and during phase 2 an emission from a larger area 2 around a higher magnetic arcade dominates. The light curves for areas 1 and 2 (Figure 2d) have their maxima shifted in time as in Figure 1d, but this time they only compose a one-peak light curve, as also seen in the GOES record (Figure 2e).

We investigated nine flare hybrids that were well observed by Yohkoh (Table 1). In each case the evolution in the SXT images was very similar, namely during phase 1 the emission was concentrated in a system of rather small loops, and during phase 2 the emission came 

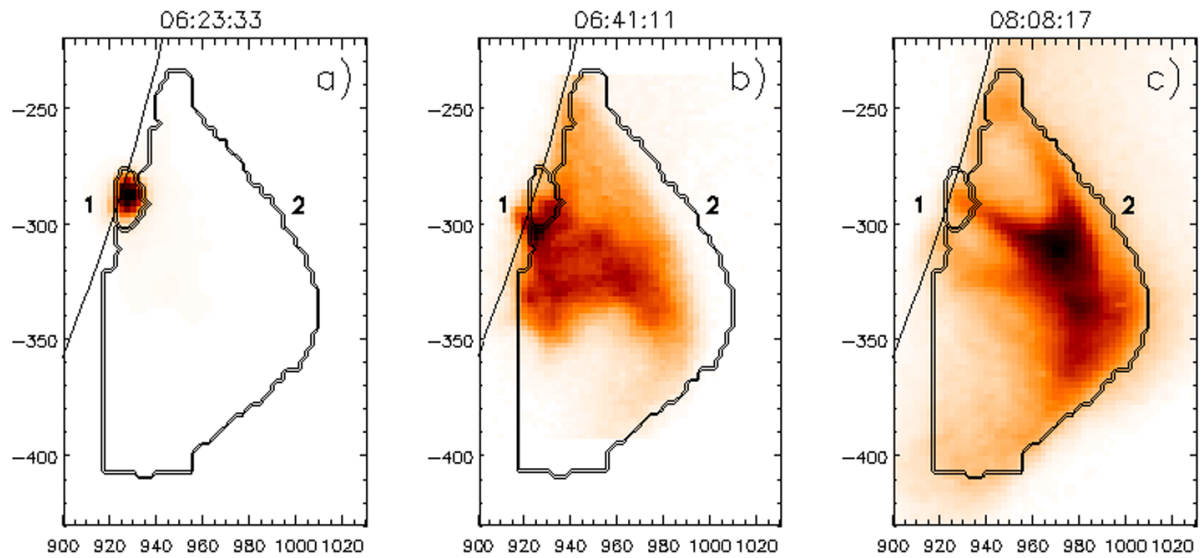

SXT/AIMg flwx

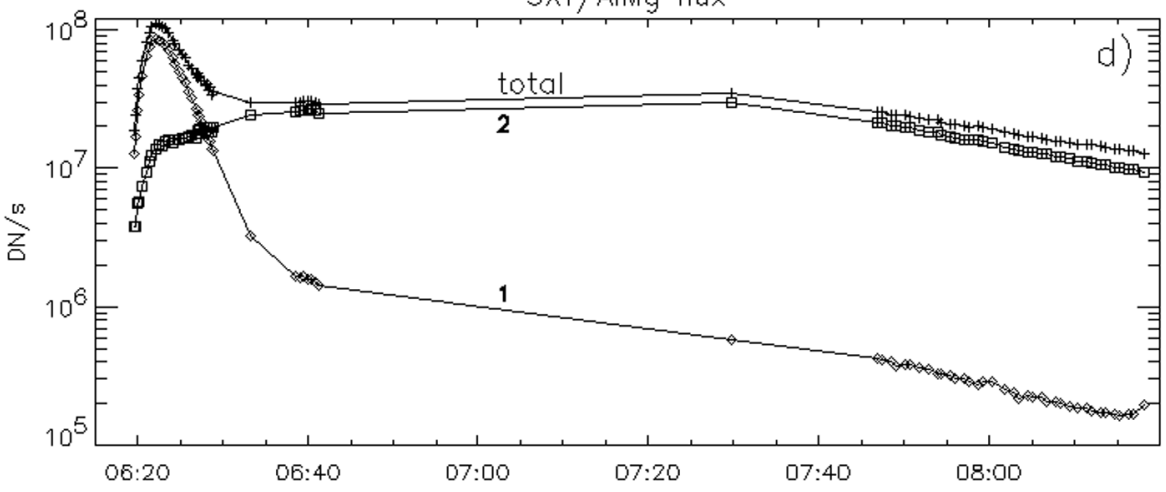

GOES X-Rays:

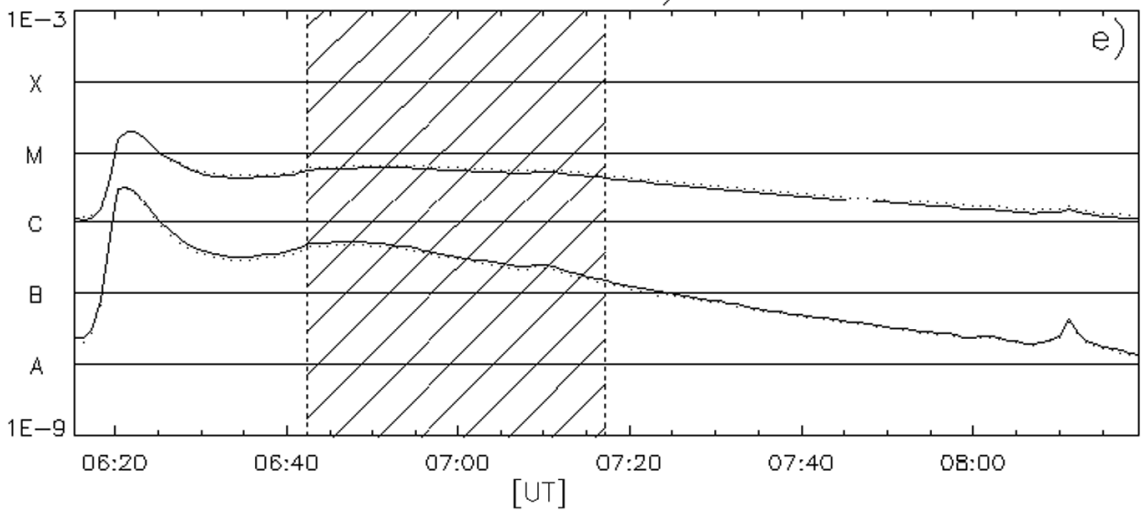

Figure 1 a) -c) The SXT/AlMg images of the flare hybrid SOL1992-11-05T06:22 (M2.0). The intensity scale is reversed. The solid line shows the solar limb, the double solid lines show the border of areas 1 and 2 which brightened in phase 1 and 2, respectively. d) The SXT/AlMg light curves for areas 1 (diamonds), 2 (squares), and the total signal (crosses). e) The GOES light curves (upper curve $-1-8 \AA$ range, lower curve $-0.5-4 \AA$ range). The hatched areas show the Yohkoh satellite nights. 

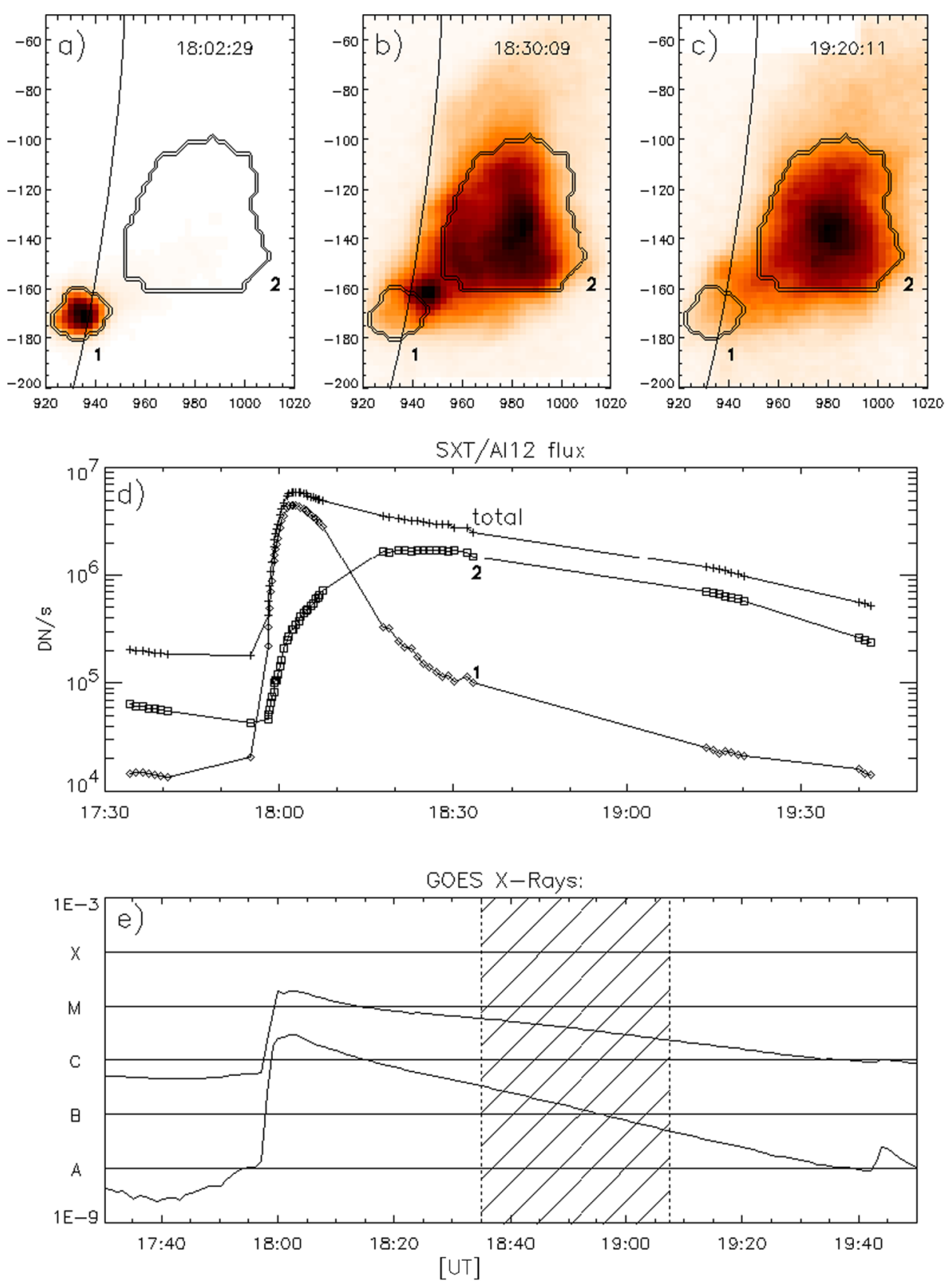

Figure 2 a) -c) The SXT/A112 images of the flare hybrid SOL1992-09-09T18:03 (M1.9). d) The SXT/Al12 light curves for areas 1, 2, and the total signal. e) The GOES light curves. For detailed explanations, see caption to Figure 1.

from a larger arcade of loops. However, only the flare hybrid from Figure 1 had a doublepeaked GOES light curve, while for the other events, GOES recorded light curves with a single peak. Thus, we conclude that an intrinsic feature of a flare hybrid GOES light curve 
Table 1 List of investigated flare hybrids.

\begin{tabular}{llllll}
\hline No. & Date & $\begin{array}{l}\text { Max. time } \\
{[\mathrm{UT}]}\end{array}$ & $\begin{array}{l}\text { GOES } \\
\text { class }\end{array}$ & Coordinates & NOAA AR \\
\hline 1 & 30-Jan-92 & $17: 15$ & M1.6 & S12 E84 & 7042 \\
2 & 8-Jul-92 & $09: 50$ & X1.2 & S11 E46 & 7220 \\
3 & 11-Aug-92 & $22: 28$ & M1.4 & N16 E90+ & 7260 \\
4 & 21-Aug-92 & $11: 10$ & M1.0 & N14 W40 & 7260 \\
5 & 6-Sep-92 & $09: 07$ & M3.3 & S11 W38 & 7270 \\
6 & 9-Sep-92 & $18: 03$ & M1.9 & S11 W78 & 7270 \\
7 & 5-Nov-92 & $06: 22$ & M2.0 & S18 W90+ & 7323 \\
8 & 9-Oct-93 & $08: 11$ & M1.1 & N11 W78 & 7590 \\
9 & 22-Sep-97 & $14: 16$ & C4.7 & S28 E43 & 8088 \\
\hline
\end{tabular}

is a rather strong asymmetry consisting of by a short rise typical for flares of class 1 followed by a slow decay typical for flares of class 2 . For events 1 to 6,8 , and 9 from Table 1 we found that the rise phase was 5 to 20 times shorter than the decay phase.

We used SXT data to calculate values of some parameters averaged over two systems of loops that form the investigated flare hybrids. For this aim we used the filter ratio method (Hara et al., 1992) employing the Be119 and Al12 image pairs as the first choice or the Al12 and Al.1 image pairs when the Be119 images were not available. In this way, we obtained a set of values characterizing the evolution of the temperature, $T$, and emission measure, $E M$. We estimated the total volume, $V$, of the two components, hence values of the electron density number, $N_{\mathrm{e}}=\sqrt{E M / V}$, became available. Next, the total thermal energy, $E_{\mathrm{th}}=$ $3 N_{\mathrm{e}} k_{\mathrm{B}} T V$ was calculated, where $k_{\mathrm{B}}$ is the Boltzmann constant. Finally, the heating rate per unit volume, $E_{\mathrm{H}}=\left(\mathrm{d} E_{\mathrm{th}} / \mathrm{d} t\right)+E_{\mathrm{C}}+E_{\mathrm{R}}$, was calculated, where $E_{\mathrm{C}}$ are the conductive losses and $E_{\mathrm{R}}$ are the radiative losses.

In Table 2 the highest values for these parameters are presented. Moreover, the Be119 light-curve parameters are summarized in Columns 2 to 4, where the time of maximum, the strongest signal, and its full width half maximum (FWHM) are given, respectively. We estimate that the typical values of the relative errors are lower than $2-3 \%$ for the intensity, temperature, and emission measure. For the volume and the related parameters the relative errors are definitely larger, about 15 to $25 \%$. In each case, large amounts of energy were released in the two flare hybrid components. The relations between parameters characterizing phase 1 and phase 2 of particular flare hybrids can be different, but some trends are seen. Phase 1 always occurs in a smaller magnetic structure than phase 2 and lasts not as long. The smaller volume involved in phase 1 leads to its larger electron number density, higher heating rate, and lower total thermal energy than phase 2. Compare the mean values and their standard deviations given in the bottom rows of Table 2.

Figures $1 \mathrm{a}-\mathrm{c}$ and $2 \mathrm{a}-\mathrm{c}$ show that the areas labeled 1 are situated near footpoints of arcades 2. Moreover, the light curves in Figures $1 \mathrm{~d}$ and $2 \mathrm{~d}$ show that the signal started to rise in arcades 2 at the beginning of phase 1, not seen in Figures 1a and 2a, which are scaled to the brightest pixel. These facts strongly support the scenario that the reported flares were not coincidences of two independent events; one of class 1, another of class 2, but were instead the result of an interaction between the loop systems 1 and 2. We have observed similar behaviors for other investigated flare hybrids.

A fundamental question arises. Is phase 2 caused by an additional energy release in the arcade, or is it an effect of the long plasma cooling time within the larger structure? There is no doubt that magnetic reconnection between loop systems 1 and 2 can heat both 


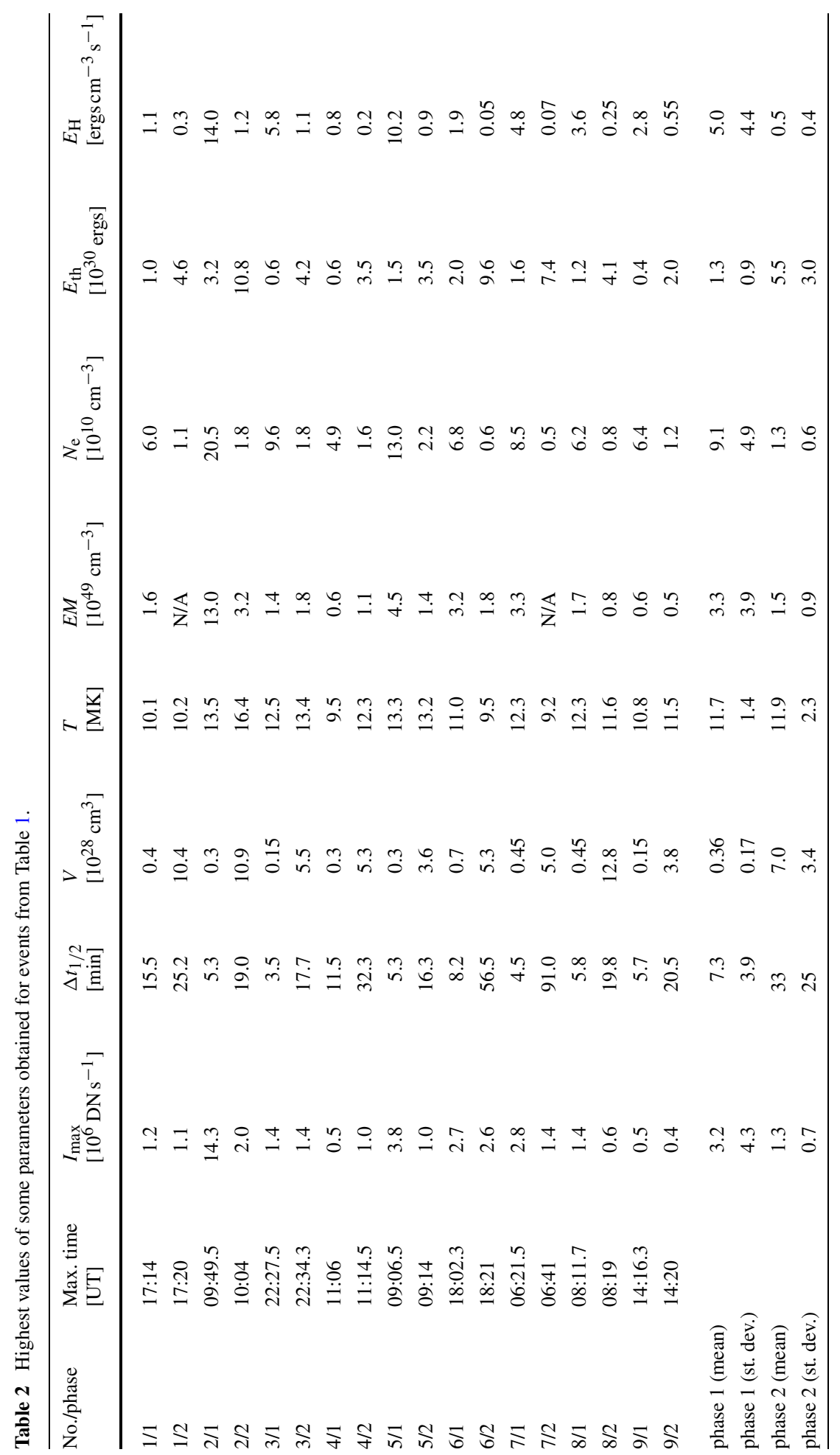


systems. The occurrence of magnetic of reconnection is supported by intense HXR emission occurring during phase 1, when SXR emission strongly rises in both systems. There is no doubt either that conductive and radiative losses are higher in system 1 than in system 2 as a result of smaller sizes and higher electron number densities, respectively (see Table 2). The higher energy losses should justify the shorter evolution timescales for system 1 in comparison with system $2, \tau_{1} \ll \tau_{2}$. However, the real evolution of flare loops is a complex interplay between heating and cooling processes.

Jakimiec et al. (1992) introduced the density-temperature $\left(N_{\mathrm{e}}-T\right)$ diagram as a very useful diagnostic tool to determine heating process in a single flaring loop based on SXR observations. They showed that flare evolutionary paths during the decay phase in this diagram strongly depend on the duration of the energy release. When the heating is switched off abruptly, a cooling due to conductive and radiative losses quickly decreases the temperature and causes a steep slope of the path of $\sim 2$. When the decay of the heating rate is rather slow, the cooling is much slower and the slope of the path is $\sim 0.5$.

We investigated evolutionary paths of the analyzed flare hybrids on the $\sqrt{E M}-T$ diagram. When we built the paths with the data from the whole flare area, the paths were more complex than those obtained for simple hydrodynamic flare models (Jakimiec et al., 1992). Sylwester et al. (1993) interpreted the complex evolutionary paths as a consequence of involving a set of distinct loops within the same flaring structure. The separate evolutionary paths on the $\sqrt{E M}-T$ diagram of the two components of flare hybrids resemble a path modeled hydrodynamically for a single loop. Moreover, the decay slopes for components 1 and 2 usually are very similar, suggesting a slow decay of the heating rate. We stress that the same phases in the evolutionary paths are shifted in time, for example, when we observe signatures of a prolonged energy release in system 2 (a slope of the path $\sim 0.5$ ), the evolutionary path for system 1 is completed.

Unfortunately, phase 2 in the investigated flare hybrids lasted long enough to be interrupted by the satellite night. Further observations made during the next orbit in the late decay phase of flares suffered from a lack of Be119 images, which prevented a good temperature and emission measure diagnostics. For these reasons we cannot be sure that phase 2 of the investigated flare hybrids is caused by an additional energy release in system 2. Moreover, the available images do not allow us to identify the place of an additional reconnection.

\subsection{Hard X-Rays}

The HXR light curves in four energy channels for the flare in Figure 1 (5 November 1992) are given in Figure 3. They were recorded by the Hard X-ray Telescope (HXT: Kosugi et al., 1991) onboard the Yohkoh satellite. In all the channels a sequence of almost equally spaced pulses, $P \approx 13 \mathrm{~s}$, is seen. At lower energies (below $33 \mathrm{keV}$ ), this sequence lasts for about three minutes, between 06:19 and 06:22 UT, while at higher energies (above $33 \mathrm{keV}$ ), the pulses can only be detected above the background between 06:19 and 06:20 UT because of lower count statistics. Similar pulsations, called quasi-periodic pulsations (QPP), are observed in many solar flares, and it is commonly accepted that they are caused by MHD oscillations excited in flaring magnetic structures (Nakariakov and Melnikov, 2009, and references therein).

The pulses are modulated by an additional gradual component, which is well observed below $33 \mathrm{keV}$ and absent above $53 \mathrm{keV}$. It consists of two broad enhancements lasting at least two minutes each, the maxima of which are separated in time by about $90 \mathrm{~s}$ (06:19:50 and 06:21:20 UT, respectively). The hardness ratio of a net signal above the background for two successive channels proves that the photon energy spectra of pulses are harder than the spectra of the gradual component. 
Figure 3 The Yohkoh hard $\mathrm{X}$-ray (HXR) light curves in four energy ranges. The number of counts are averaged per second and per subcollimator.
5 NOV 1992 FLARE
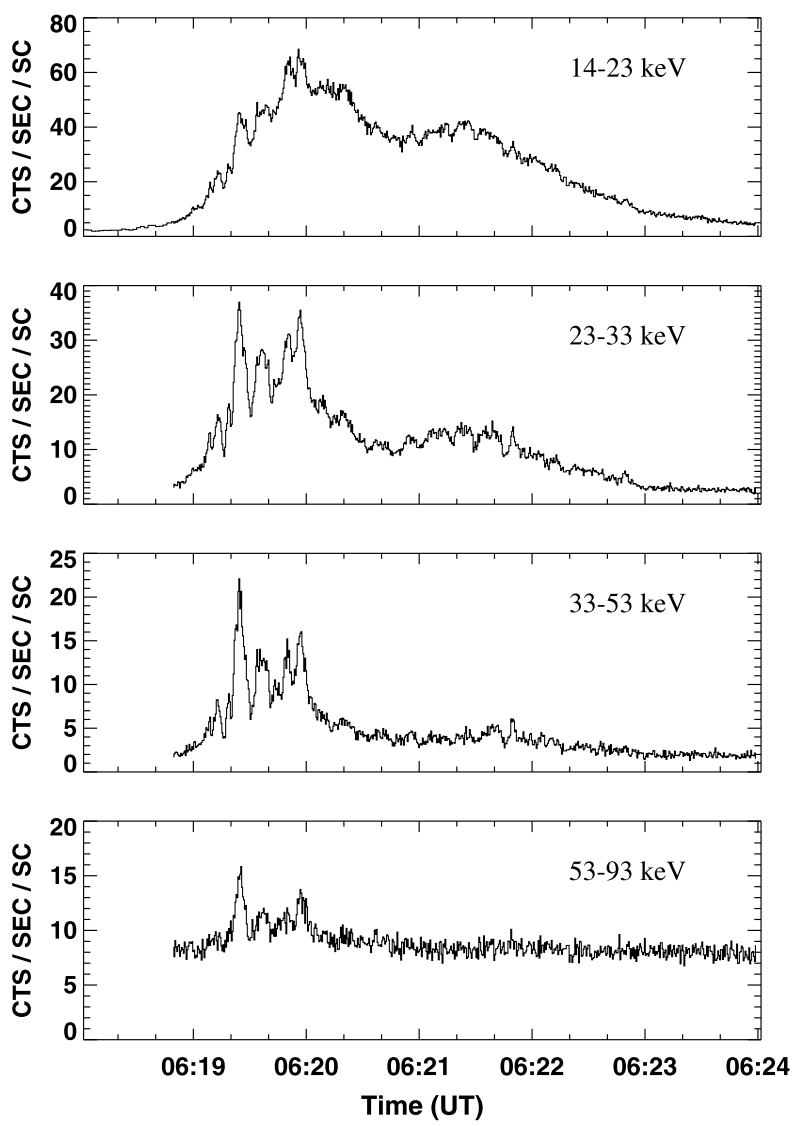

Recently, Tomczak and Szaforz (2014) analyzed the similar solar flare of 2 October 2001 (SOL2001-10-02T17:31, C4.7), in which more energetic pulses with a period $P_{1}=26-31 \mathrm{~s}$ were modulated by three more gradual enhancements with a period $P_{2}=110 \mathrm{~s}$. The authors found that these periods were excited simultaneously in a flare hybrid by an interaction between a system of small loops and a high arcade of loops. They also proved that the shorter period was excited in the small loops and the longer period in the arcade.

The observations available for the flare of 5 November 1992 do not allow us to spatially separate the two periods, but the close similarity of the overall magnetic configuration with the flare of 2 October 2001 suggests an interaction between small loops and a high arcade excited simultaneously by MHD oscillations in both magnetic structures. We would like to stress that in some articles reporting the presence of QPP with two distinctly different periods excited simultaneously in HXRs, an interaction between two magnetic structures of different sizes is always mentioned, see e.g. Asai et al. (2001), and Nakariakov et al. (2006).

We investigated HXR light curves of all the flare hybrids from Table 1 looking for similarities with the flare of 5 November 1992, i.e. showing two distinctly different periods excited simultaneously. Unfortunately, some light curves were not complete due to the passage of the satellite through the South Atlantic Anomaly, therefore we incorporated the available light curves derived by the Burst and Transient Source Experiment (BATSE: Fishman et al., 1992) on board the Compton Gamma Ray Observatory. In summary, we confirm 
the presence of QPPs for seven flare hybrids, but only for three events two distinctly different periods were excited simultaneously. Apart from the flare of 5 November 1992, these were flares of 8 July 1992, 21 August 1992, and 9 September 1992. Four events from nine are for sure too few to claim that QPPs with two distinctly different periods excited simultaneously are intrinsic features of flare hybrids in HXRs. Nevertheless, this characteristic HXR pattern suggests an influence of the magnetic configuration of flare hybrids, in which two interacting magnetic structures have distinctly different sizes.

\subsection{Extreme-Ultraviolet}

Taking advantage of the new instruments the Atmospheric Imaging Assembly (AIA: Lemen et al., 2012), and the Extreme-ultraviolet Variability Experiment (EVE: Woods et al., 2012) onboard the NASA Solar Dynamics Observatory (SDO), Woods et al. (2011) introduced a new class of flares, called EUV late-phase (ELP) flares. Their defining feature is an additional peak of the coronal emission seen in the spectral line of Fe XVI $335 \AA$ Accurring half an hour to two hours after the GOES SXR peak. This line is an indicator of plasma with a temperature of about $3 \mathrm{MK}$. For other EUV spectral lines use for detecting the warm plasma, e.g. Fe XVIII $94 \AA$ ( $\sim 6 \mathrm{MK})$ or Fe XV $284 \AA$ ( $\sim 2 \mathrm{MK})$, the late peaks also occur (Sun et al., 2013). Woods (2014) specified other features of ELP flares: (1) no significant counterpart of the late peak in the hot plasma (the GOES SXR or Fe XX/Fe XXIII $133 \AA$ ), (2) an eruption followed by a coronal dimming seen in the cooler plasma, e.g. Fe IX $171 \AA$, preceding the late peak, and (3) a different system of longer loops visible above the place where the peak of the hot plasma was emitted.

These features of ELP flares strongly resemble those presented in Section 2.1 for flare hybrids observed in SXRs. The evolution of ELP flares consists of the two following phases: the first, when the emission is concentrated in a small system of loops (system 1), and the second, when an additional system of longer loops (system 2) occurs and its emission dominates. Moreover, an interaction between the two systems undoubtedly exists. Therefore we consider the ELP flares simply as a new face of flare hybrids that we can investigate thanks to the new EUV instruments onboard the SDO. A broad wavelength range and high temporal, spatial, and spectral resolutions allow us to measure changes in many EUV spectral lines that are indicators of plasma in a wide range of temperatures. This gives us the opportunity to decide whether additional energy release or a long timescale of plasma cooling is responsible for the prolonged evolution.

In several case studies of ELP flares (Hock et al., 2012; Liu et al., 2013; Dai, Ding, and Guo, 2013; Sun et al., 2013), the authors investigated sequences of AIA light curves ordered with decreasing temperature of the filters from Fe XX/Fe XXIII $133 \AA$ ( $\sim 10$ MK) to Fe IX $171 \AA(\sim 0.7 \mathrm{MK})$. The signal was formed by selected fragments of ELP flares. The authors agree that during phase 1 in system 1 of the investigated flares the maximum is shifted in time, first occurring for the hot plasma, later on for the warm plasma, and finally for the cold plasma. This appearance is interpreted as a consequence of plasma cooling due to radiative and conductive losses. Liu et al. (2013) reported a similar delay during phase 2 in system 2. They obtained timescales definitely longer than for phase 1 as a result of fewer conductive (larger loops) and radiative (lower electron density number) losses. Sun et al. (2013) reported similar results, but only for the hot and warm plasma. The evolution of the cold plasma was more complex.

On the other hand, Dai, Ding, and Guo (2013) obtained a more complicated picture, in which each light curve for system 2 during phase 2 shows several maxima. They interpreted this observations as a proof of several episodes of energy release in this system. Hock et al. 
(2012) noted a lack of hot plasma in system 2 during phases 1 and 2 and interpreted this as a proof of additional energy release in system 2 causing only a modest increase of the temperature. The same interpretation can be used to explain the double maxima visible in light curves of cold filters in the flare analyzed by Sun et al. (2013).

Recently, Li et al. (2014) have published additional arguments supporting a twofold explanation of phase 2 in ELP flares. They modeled the EUV emission from sets of loops having different lengths and different heating rates using the enthalpy-based thermal evolution of loops (EBTEL) model (Klimchuk, Patsourakos, and Cargill, 2008). They found that two separate maxima in the Fe XVI $335 \AA$ can be modeled by simultaneous heating during phase 1 in two distinct loops of different lengths and by repeating the heating during phase 2 in system 2. Li et al. (2014) pointed out the importance of the AIA UV $1600 \AA$ channel to distinguish between cooling and heating for the ELP flares. Some contribution to the emission in this channel comes from the C IV line formed in the upper chromosphere. Therefore the AIA UV $1600 \AA$ channel is very sensitive to flare energy release. Indeed, the light curves presented by Li et al. (2014) support the interpretation of additional energy release for the flares investigated by Dai, Ding, and Guo (2013) and Sun et al. (2013) as well as a lack of energy release in phase 2 of flares investigated by Liu et al. (2013).

\section{How Common Are Flare Hybrids?}

We have investigated the occurrence of flare hybrids between September 1991 and April 1999. For this aim we analyzed SXR GOES light curves and qualified as flare hybrids (1) those showing a double maximum, under the condition that both maxima occurred in the same active region, or (2) those having a strongly asymmetric light curve, i.e. a fast rise and a slow decay. If possible, doubtful events were verified on the basis of SXT images. In summary, we identified 577 flare hybrids. Altogether, 15178 flares occurred in the investigated time interval. This translates into a $3.8 \%$ contribution of flare hybrids.

Figure 4 presents the number of flare hybrids for each trimester in the investigated time interval. The total number of all flares is also given for comparison. The number of flare hybrids roughly mimics the solar cycle phase. However, the contribution of this class in the full population was the highest around the activity minimum $(1995-1997) \approx 9-13 \%$, whereas during enhanced activity $(1991,1993,1998-1999)$ it decreased to $\approx 2-4 \%$.

Recently, a more comprehensive statistical research of ELP flares has been reported by Woods (2014). He investigated SXR GOES light curves from 1974 to 2013 and searched

Figure 4 a) Number of flare hybrids (red bins) and all flares (black bins) that occurred between September 1991 and April 1999. The size of a bin is four months. b) Ratio of flare hybrids for each trimester bin.
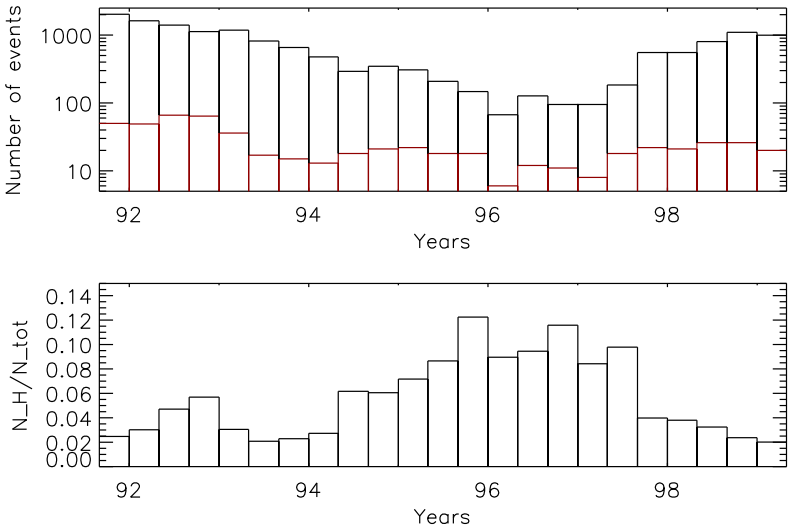
for a dual-decay behavior, i.e. a steep slope followed by a moderate slope. He argued that the first slope represents cooling of shorter loops (system 1), whereas the second slope represents the cooling of longer loops (system 2). Woods (2014) obtained that the contribution of flares showing dual-decay is $7.9 \%$, in particular, from 2010 to 2013 it was $10.5 \%$. He found that the contribution of ELP flares is the highest $(20-30 \%)$ around a solar-cycle minimum. He also found that the higher the flare class, the higher the contribution of the dual-decay flares. The very important work of Woods (2014) aimed at validating the results obtained with SDO/EVE and AIA data. He reported that $36 \%$ and $57 \%$ of flares showing double-decay from 2010 to 2011 and from 2011 to 2013, respectively, do not show features that would allow us to classify them as ELP flares.

In spite of fundamental differences between the methodologies used by Woods and us, there are also some similarities. The most striking is that in both studies the highest frequency occurs during low solar activity. The reason for this seems to be rather trivial. During low activity, flares are not as frequent, thus their GOES light curves do not overlap, and every selection criterion works perfectly. Keeping in mind that during higher activity the probability of occurrence of active regions with complex magnetic structure with loops of different lengths is even higher, the contribution of $9-13 \%$ can be treated as a lower limit of flare hybrids. It is interesting that the frequency values of 20-30\% given by Woods (2014) for the low activity phase, after adopting his $43 \%$-validation obtained for events from 2011 to 2013 , decrease to $9-13 \%$.

Woods et al. (2011) noted that ELP flares show a tendency of occurring within the same active regions. For example, their Table 2 shows that of the 22 ELP flares, six events occurred in NOAA AR 11069 and another six in NOAA AR 11121. We did not complete the list of flare hybrids including the active region identification, but during the investigation of some active regions within which a flare hybrid occurred, we found that the same magnetic configuration was flaring several times. For example, the flare hybrid described by Tomczak and Szaforz (2014) was preceded by three other flare hybrids that occurred in the same active region NOAA AR 9628.

\section{Magnetic Configuration}

All the available observations of flare hybrids strongly suggest the existence of two sets of magnetically related loop systems. This means a multipolar magnetic configuration, in which magnetic reconnection plays a crucial role in energy release and in shaping a new configuration that becomes more potential. In previous studies the following particular configurations have been proposed: a classical quadrupolar topology based on breakout reconnection (Hock et al., 2012), an asymmetric quadrupolar topology with a sigmoidal core (Liu et al., 2013), a multistep reconnection in a multipolar topology (Dai, Ding, and Guo, 2013), and a fan-spine topology (Sun et al., 2013).

We usually conclude about the reconnection indirectly by observing the emission of nonthermal electrons in HXRs and the emission of the multithermal plasma in SXRs and EUV. Sometimes eruptions can be treated as a signature of the reconnection, and expanding loops can even initiate a following reconnection with a higher magnetic system (Su et al., 2012; Dai, Ding, and Guo, 2013). Occasionally, it is possible to identify the loops that are the product of reconnection, e.g. see Figure 4 in Sun et al. (2013). Tomczak (2013) reported a unique flare hybrid (SOL2001-10-02T17:31, C4.7), in which the reconnection occurred between a new emerging flux and an overlying coronal field. In SXR images recorded by the SXT the whole process is seen very clearly, starting from a fast expansion of emerging loops 
and their evident deformation in the vicinity of the reconnection site, followed by vigorous plasma motions inside the reconfiguring loops and the formation of a new system of loops.

The emerging flux model (Heyvaerts, Priest, and Rust, 1977), in which subphotospheric magnetic fields emerge as a result of buoyancy within an already existing active region and meet overlying coronal magnetic fields easily explains the main characteristics of flare hybrids. Continuous emergence of a new flux under a stable magnetic environment can produce homologous flares occurring in the same location with similar morphologies. The examples mentioned in Section 3 are most likely the illustration of this process, explaining the tendency of flare hybrids to occur in the same active region.

\section{Conclusions and Prospects}

Our intention is to recall the forgotten term introduced by the late Zdeněk Švestka many years ago. His experience and intuition suggested the importance of flare hybrids that show a complex evolution in which their appearance changes completely. Events like these warn us against general conclusions drawn from a limited set of observations. The conclusion can be incorrect even though it agrees with the available data. A closer insight sometimes requires a detailed study of the evolution of the active region in which the investigated event occurred.

For the past 25 years, an abundance of new data has been obtained by successive solar satellites. On this basis, the following typical observational features of flare hybrids are easily recognized: (1) separate systems of loops seen in EUV and SXR images, (2) doublepeaked or strongly asymmetrical light curves in these wavelengths, (3) multiperiodicity of pulses recorded in HXRs. Now it is possible to give comprehensive answers to the general questions asked by Švestka (1989).

The defining condition for the occurrence of a flare hybrid seems to be the reconnection between two systems of magnetic loops, a system 1 and a system 2, one shorter, the other longer. This means that the condition necessary for the occurrence is a multipolar magnetic configuration. The process can be initiated, for example, by a new magnetic flux (system 1) emerging within the already existing active region (system 2). The reconnection triggers energy release and the chromospheric evaporation, which fills both systems with plasma and initiates intense SXR and EUV emissions. System 1 is quickly cooled by strong radiative and conductive losses, completing phase 1 . Further evolution of flare hybrids (phase 2) is connected with the evolution of system 2. The prolonged SXR and EUV emissions in this system might be the effect of the long timescale of plasma cooling due to fewer radiative and conductive losses. However, in some events proofs of additional energy release in system 2 are also given. A new reconnection site can be somehow connected with eruptions observed in phase 1 . The amount of energy released in system 2 during phase 2 establishes the highest temperature of the plasma and in this way, the accessibility of observations in different SXR and EUV filters.

It is easier to recognize typical features of flare hybrids when the differences between the lengths of the interacting systems of loops are greater. However, even very different systems do not always cause the clear features of these events. The frequency estimates of flare hybrids presented in Section 3 should therefore be treated as a lower limit of the actual value.

Flares are important for space weather because they produce photons that are energetic enough to quickly enhance the ionization in Earth's upper atmosphere. Integration of emitted flux over wavelengths shows the importance of the EUV flux. The prolonged EUV emission 
makes the flare hybrids extremely geoeffective through its prolonged impact on the Earth's atmosphere. For this reason, new methods introducing an early warning of an occurrence of these events would be useful. Future complex investigation including X-ray and EUV observations should verify, for example, the usefulness of the multiperiodicity in HXRs for predicting the EUV late maximum.

Acknowledgements The Yohkoh satellite is a project of the Institute of Space and Astronautical Science of Japan. We are very thankful to the referee for important comments that helped to improve this article. We acknowledge financial support from the Polish National Science Centre grant 2011/03/B/ST9/00104.

Open Access This article is distributed under the terms of the Creative Commons Attribution 4.0 International License (http://creativecommons.org/licenses/by/4.0/), which permits unrestricted use, distribution, and reproduction in any medium, provided you give appropriate credit to the original author(s) and the source, provide a link to the Creative Commons license, and indicate if changes were made.

\section{References}

Asai, A., Shimojo, M., Isobe, H., Morimoto, M., Yokoyama, T., Shibasaki, K., et al.: 2001, Astrophys. J. 562, L103. DOI.

Dai, Y., Ding, M.D., Guo, Y.: 2013, Astrophys. J. 773, L21. DOI.

Fishman, G.J., Meegan, C.A., Wilson, R.B., Paciesas, W.S., Pendleton, G.N.: 1992, In: Shrader, C.R., Gehrels, N., Dennis, B. (eds.) The Compton Observatory Science Workshop, NASA CP 3137, 26.

Hara, H., Tsuneta, S., Lemen, J.R., Acton, L.W., McTiernan, J.M.: 1992, Publ. Astron. Soc. Japan 44, L135.

Heyvaerts, J., Priest, E.R., Rust, D.M.: 1977, Astrophys. J. 216, 123. DOI.

Hock, R.A., Woods, T.N., Klimchuk, J.A., Eparvier, F.G., Jones, A.R.: 2012. arXiv.

Jakimiec, J., Sylwester, B., Sylwester, J., Serio, S., Peres, G., Reale, F.: 1992, Astron. Astrophys. $253,269$.

Klimchuk, J.A., Patsourakos, S., Cargill, P.J.: 2008, Astrophys. J. 682, 1351. DOI.

Kosugi, T., Makishima, K., Murakami, T., Sakao, T., Dotani, T., Inda, M., et al.: 1991, Solar Phys. $136,17$. DOI.

Lemen, J.R., Title, A.M., Akin, D.J., Boerner, P.F., Chou, C., Drake, J.F., et al.: 2012, Solar Phys. $275,17$. DOI.

Li, Y., Ding, M.D., Guo, Y., Dai, Y.: 2014, Astrophys. J. 793, 85. DOI.

Liu, K., Zhang, J., Wang, Y., Cheng, X.: 2013, Astrophys. J. 768, 150. DOI.

Nakariakov, V.M., Melnikov, V.F.: 2009, Space Sci. Rev. 149, 119. DOI.

Nakariakov, V.M., Foullon, C., Verwichte, E., Young, N.P.: 2006, Astron. Astrophys. 452, 343. DOI.

Pallavicini, R., Serio, S., Vaiana, G.: 1977, Astrophys. J. 216, 108. DOI.

Švestka, Z.: 1989, Solar Phys. 121, 399. DOI.

Su, Y., Dennis, B.R., Holman, G.D., Wang, T., Chamberlin, P.C., Savage, S., et al.: 2012, Astrophys. J. 746, L5. DOI.

Sun, X., Hoeksema, J.T., Liu, Y., Aulanier, G., Su, Y., Hannah, I.G., et al.: 2013, Astrophys. J. 778, 139. DOI.

Sylwester, B., Sylwester, J., Serio, S., Reale, F., Bentley, R.D., Fludra, A.: 1993, Astron. Astrophys. $267,586$.

Tomczak, M.: 2013, Cent. Eur. Astrophys. Bull. 37, 585.

Tomczak, M., Szaforz, Ż: 2014, Cent. Eur. Astrophys. Bull. 38, 111.

Tsuneta, S., Acton, L., Bruner, M., Lemen, J., Brown, W., Caravalho, R., et al.: 1991, Solar Phys. $136,37$. DOI.

Woods, T.N.: 2014, Solar Phys. 289, 3391. DOI.

Woods, T.N., Hock, R., Eparvier, F., Jones, A.R., Chamberlin, P.C., Klimchuk, J.A., et al.: 2011, Astrophys. J. 739, 59. DOI.

Woods, T.N., Eparvier, F.G., Hock, R., Jones, A.R., Woodraska, D., Judge, D., et al.: 2012, Solar Phys. 275, 115. DOI. 